\title{
Interactive comment on "Spatial and temporal Trend Analysis of Long Term rainfall records in data-poor catchments with missing data, a case study of Lower Shire floodplain in Malawi for the Period 1953-2010" by Rabee Rustum et al.
}

\section{Rabee Rustum et al.}

r.rustum@hw.ac.uk

Received and published: 3 December 2017

Thank you very much for the careful review and valuable comments to the initial submission. We will address the comments raised on the initial submission and we will revise the manuscript accordingly to accommodate these comments. 